\title{
Three-dimensional scanning of the cornea by using a structured light module
}

\section{Digitalización tridimensional de la superficie de la córnea utilizando un módulo de luz estructurada}

\author{
J. Barrios ${ }^{1,2,3 *}$, M. Morón 1 , C. Barrios², R. Contreras ${ }^{2,3}$, A. González 3 , J. Meneses ${ }^{3}$ \\ 1. Grupo Interdisciplinario de Investigaciones Epidemiológicas en el Sistema Visual, Universidad Santo \\ Tomás, Carrera 18 No. 9-27, Bucaramanga, Santander, Colombia \\ 2. Grupo de Investigación en Ciencias Básicas y Aplicadas, Universidad Santo Tomás, Carrera 18 No. 9-27, \\ Bucaramanga, Santander, Colombia \\ 3. Grupo de Óptica y Tratamiento de Señales, Universidad Industrial de Santander, Carrera 27 Calle 9, \\ Bucaramanga, Santander, Colombia \\ (*) E-mail: juan.barrios@ustabuca.edu.co \\ Received: 07/12/2016 Accepted: 18/08/2017 \\ DOI: $10.7149 /$ OPA.50.4.49067
}

\begin{abstract}
:
Corneal topography is a computerized diagnostic tool that creates a three-dimensional map of the surface of the cornea. Corneal topography provides tools needed to know the morphology, optics, power distribution and radii of the anterior surface of the cornea. This is of great help for multiple fields of optometry and ophthalmology: contact lens fitting, keratoconus detection and tracking and corneal ecstasies, detection of suspicious corneas in refractive surgery, etc. Although there are basically two types of corneal surveyors: specular reflection and elevation, many authors agree that the accuracy of these devices is affected by multiple factors, which obey both the mathematical process used and certain physiological variables. Three-dimensional (3D) scanning systems, consisting of devices based on a structured light module, are widely used to perform high-precision measurements in primarily industrial and medical applications. The present work is proposed to show the application of fringe projection technique in three-dimensional scanning of corneal surface.
\end{abstract}

Key words: Three-dimensional scanning, corneal surface, optometry

\section{RESUMEN:}

La topografía corneal es una herramienta diagnóstica computarizada que crea un mapa tridimensional de la superficie de la córnea. La topografía corneal proporciona herramientas necesarias para conocer la morfología, la óptica, la distribución de la potencia y de los radios, de la superficie anterior de la córnea. Esto es de gran ayuda para múltiples campos de la optometría y la oftalmología: adaptación de lentes de contacto, detección y seguimiento de queratocono y ectasias corneales, detección de córneas sospechosas en cirugía refractiva, etc. Aunque existen básicamente dos tipos de topógrafos corneales: de reflexión especular y de elevación, muchos autores coinciden que la precisión de estos dispositivos es afectada por múltiples factores, que obedecen tanto al proceso matemático empleado como a ciertas variables fisiológicas. Los sistemas de digitalización tridimensional (3D), formados por dispositivos que se basan en un módulo de luz estructurada, son usados ampliamente para realizar mediciones de alta precisión en aplicaciones industriales y médicas principalmente. El presente trabajo se plantea con el objetivo de mostrar la aplicación de la técnica de proyección de franjas en la digitalización tridimensional de la superficie de la córnea.

Palabras clave: Digitalización tridimensional, superficie de la córnea, optometría 


\section{REFERENCES AND LINKS / REFERENCIAS Y ENLACES}

[1] D. R. Iskander, P. Wachel, P. N. Simpson, A. Consejo, D. A. Jesus, "Principles of operation, accuracy and precision of an Eye Surface Profiler," Ophthalmic and Physiological Optics, 36(3), 266-278 (2016).

[2] E. Kreyszig, Differential Geometry. New York: Dover Publications (1991).

[3] Y. Mejía-Barbosa, D. Malacara-Hernández, "A review of methods for measuring corneal topography," Optometry \& Vision Science, 78(4), 240-253 (2001).

[4] F. Karimian, S. Feizi, A. Doozandeh, A. Faramarzi, M. Yaseri, "Comparison of corneal tomography measurements using Galilei, Orbscan II, and Placido disk-based topographer systems," Journal of refractive surgery, 27(7), 502-508 (2011).

[5] F. Vivó Sánchez, J. L. Garrido Tundidor, “Topografía corneal mediante discos de Plácido. Estrategias de optimización de la estimación topográfica," Gaceta de optometría y óptica oftálmica, 457, 24-29 (2011).

[6] H. Liang, E. Olesch, Z. Yang, F. Schiffers, G. Häusler, "Single-Shot Phase Measuring Deflectometry for Cornea Measurement," Advanced Optical Technologies, 5(5-6), 433-438 (2016).

[7] G. Savini, M. Carbonelli, A. Sbreglia, P. Barboni, G. Deluigi, K. J. Hoffer, "Comparison of anterior segment measurements by 3 Scheimpflug tomographers and 1 Placido corneal topographer," Journal of Cataract \& Refractive Surgery, 37(9), 1679-1685 (2011).

[8] W. A. Douthwaite, A. Parkinson, "Precision of Orbscan II assessment of anterior corneal curvature and asphericity," Journal of refractive surgery, 25(5), 435-443 (2009).

[9] M. Belin, Tomografía corneal basada en la elevación. JP Medical Ltd (2012).

[10] W. Chen, C. McAlinden, K. Pesudovs, Q. Wang, F. Lu, Y. Feng, J. Huang, "Scheimpflug-Placido topographer and optical low-coherence reflectometry biometer: Repeatability and agreement," Journal of Cataract \& Refractive Surgery, 38(9), 1626-1632 (2012).

[11] A. L. González, C. R. Contreras, J. E. Meneses, “Accurate and flexible calibration technique for fringe projection profilometry by using encoded points and Fourier analysis," In SPIE Sensing Technology + Applications, 9110, 91100X-1 (2014).

[12] Z. Zhang, "Flexible camera calibration by viewing a plane from unknown orientations," In Computer Vision, 1999. The Proceedings of the Seventh IEEE International Conference on, 1, 666-673 (1999).

[13] R. C. Gonzalez, R. E. Woods, Digital image processing. Upper Saddle River, NJ (2002).

[14] L. Zhu, E. Liu, J. H. McClellan, “An Automatic Arrival Time Picking Method Based on RANSAC Curve Fitting," In 78th EAGE Conference and Exhibition 2016 (2016).

\section{Introducción}

En la actualidad existen diferentes técnicas que permiten realizar mediciones sobre la superficie de la córnea [1]. Asumiendo que la superficie de la córnea esté dada por $z=f(x, y)$ en un sistema cartesiano, con primeras y segundas derivadas continuas en cualquier punto, hay al menos tres formas diferentes en las que la topografía de la córnea se puede especificar:

- $\quad$ Por la superficie de elevación $f(x, y)$ respecto a una superficie de referencia conveniente. Tal superficie de referencia puede ser un plano o una esfera. Una esfera de referencia es aquella con desviación estándar mínima con respecto a la superficie de la córnea y con el mismo eje óptico. Esta es la representación más simple y da una representación directa de la superficie de la córnea.

- $\quad$ Por las pendientes locales con respecto a la superficie de referencia. En cualquier punto sobre la superficie la pendiente es una función de la dirección. Por lo tanto, la pendiente se debe especificar por dos números: su valor máximo y su dirección (gradiente). Estas pendientes se determinan matemáticamente a partir de la superficie de elevación elevación $f(x, y)$ calculando sus primeras derivadas parciales a lo largo de los ejes $x$ y $y$.

- $\quad$ Por la curvatura local. De manera general, para una superficie, la curvatura en cualquier punto depende de la dirección en la que se mide [2]. Para un punto cualquiera en la superficie, hay un valor máximo en cierta dirección y un valor mínimo en la dirección perpendicular. Con estos dos valores, es posible determinar la curvatura en cualquier dirección. Por lo tanto, para determinar la curvatura en 
cualquier dirección, se necesitan tres números, la curvatura máxima, la curvatura mínima, y la dirección correspondiente a la curvatura máxima (o mínima). Estas curvaturas se obtienen matemáticamente calculando las primeras y segundas derivadas parciales de $f(x, y)$. Asumiendo que el índice de refracción de la córnea es constante, $n=1,3375$ una representación equivalente frecuentemente utilizada por los optómetras es el poder refractivo en dioptrías dado por $D=(n-1) / R$, donde $R$ es el radio de curvatura de la superficie.

Los principios ópticos de algunos métodos que son implementados para medir la topografía de la córnea se pueden clasificar de acuerdo a los principios físicos usados en la medición [3], entre éstos se encuentran la reflexión especular, la reflexión difusa y luz dispersada. La técnica de reflexión especular considera la superficie anterior de la córnea como un espejo convexo, como en los sistemas basados en los discos de Plácido [4]. Las técnicas basadas en la reflexión de los discos de Plácido reconstruyen la superficie de la córnea a partir del análisis de la imagen reflejada sobre la cara anterior de la córnea dando lugar a distintos mapas topográficos [5]. Las técnicas de interferometría son utilizadas para evaluar elementos ópticos como lentes y espejos. En la técnica de deflectometría Moiré se analiza la deflexión de los rayos reflejados fuera de la superficie de la córnea [6]. La técnica de reflexión difusa modifica la reflexión especular de la superficie anterior de la córnea convirtiéndola en un reflector difuso, lo cual se logra aplicando fluoresceína a la película lagrimal. Con esta modificación se pueden aplicar los métodos usuales de análisis de superficies difusas, como el método de proyección de franjas [1]. La técnica de luz dispersada usa el fenómeno de dispersión de la luz cuando se propaga en un medio óptico. En este caso, el tejido de la córnea es el medio óptico donde se dispersa la luz. Parte de esta luz dispersada emerge a través de la superficie anterior de la córnea y es capturada por un sistema formador de imágenes. Se analizan los bordes de la imagen, y se obtiene información de las superficies anterior y posterior de la córnea. Esta técnica es implementada en la lámpara de hendidura. Por otra parte, entre los sistemas comerciales que son utilizados para realizar mediciones sobre la superficie de la córnea se encuentran [7]: Pentacam, Sirius, TMS-5, Keratron y Orbscan II. Tales sistemas realizan mediciones muy precisas sobre la superficie de la córnea. Por ejemplo el Orbscan II puede realizar mediciones del radio de curvatura de la córnea con una precisión de 90 micras y medidas de elevación con una precisión de 70 micras [8]. El Pentacam y el Pentacam HR son dispositivos de imagen de Scheimpflug los cuales utilizan dos cámaras. La cámara central se usa para el monitor de fijación y la medición de la pupila. La segunda, una cámara de Scheimpflug rotatoria brinda 25 o 50 imágenes de cortes seccionales durante un rastreo de menos de 2 segundos de duración. Cada imagen contiene 500 puntos de elevación con el Pentacam estándar y 2760 puntos de elevación para el Pentacam HR [9]. El sistema de topografía Sirius combina dos mecanismos de acción, una cámara doble monocromática de Scheimpflug rotatoria con la topografía de discos de Plácido. Proporciona en una sola exploración un análisis completo de la córnea y segmento anterior, incluyendo los datos de curvatura anterior y posterior de la superficie de la córnea, curvatura tangencial y axial de la superficie corneal anterior y posterior, poder refractivo corneal global, estimación biométrica de varias estructuras, mapa paquimétrico corneal total, y el mapa de frente de onda corneal. Utiliza una fuente de luz azul tipo LED de $475 \mathrm{~nm}$ para medir 35632 puntos para la superficie corneal anterior y 30000 puntos para la superficie posterior [10]. El TMS-5 usa una combinación de una cámara de Scheimpflug rotatoria y un topógrafo con discos de Plácido. El Keratron permite obtener la curvatura corneal usando el método "Arc-Step" por medio de la reflexión de sus 28 anillos para un análisis total de más de 80000 datos cubriendo el $90 \%$ de la superficie corneal. En este artículo se presenta una metodología para medir la superficie anterior de la córnea basada en un módulo de luz estructurada. Los resultados experimentales obtenidos muestran la fiabilidad y validez de la metodología desarrollada.

\section{Módulo de luz estructurada}

En la actualidad, los métodos ópticos son muy usados para digitalizar tridimensionalmente la superficie de diferentes objetos de estudio, debido a que se caracterizan por alta precisión, amplio campo de trabajo, y a que el uso de los mismos no implica contacto con la superficie del objeto. Entre estos métodos, aquellos que implementan módulos de luz estructurada han sido ampliamente estudiados y han sido aplicados en muchos campos debido a su sencillo montaje experimental, procesamiento automático y bajo costo. Un módulo de luz estructurada está formado por un proyector de video, una cámara digital y un computador que se usa para controlar la proyección del patrón de luz y la adquisición del mismo cuando se deforma sobre la superficie del objeto, ver Figura 1. El proyector es usado para proyectar una línea sobre la superficie del objeto. Debido a las variaciones topográficas de la superficie del objeto, la línea proyectada 
se distorsiona; este patrón distorsionado se captura usando la cámara digital. Después de esto, utilizando parámetros obtenidos a partir de un procedimiento de calibración que transforma, desde píxeles a unidades métricas las coordenadas de los puntos que pertenecen a la línea distorsionada, la superficie del objeto es digitalizada.

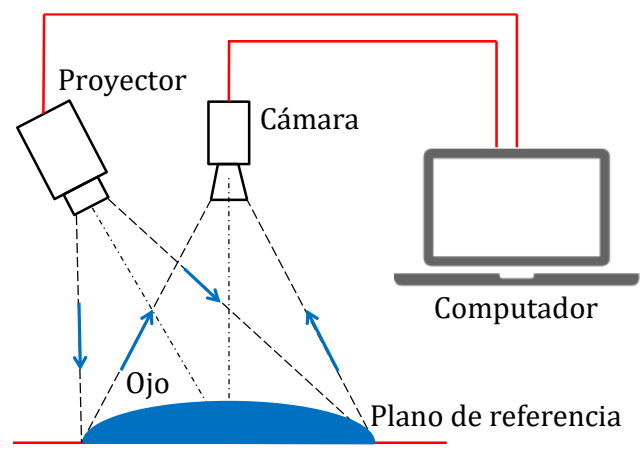

Fig.1. Módulo de luz estructurada.

\section{Sistema implementado}

El sistema que se implementó está formado por una cámara CMOS de la marca Edmund Optics, referencia E0-2013C y resolución 1600 píxeles $\times 1200$ píxeles y un proyector DELL referencia M210X, con brillo de 2000 lúmenes y resolución XGA $(1024 \times 768)$. El lente de observación de la cámara digital se fijó de tal manera que se obtuvieran imágenes de la córnea en la máxima resolución. El proyector generaba imágenes de la línea con espesor de 1 píxel ubicado a una distancia mínima de $27 \mathrm{~cm}$.

\section{Calibración del sistema}

La Figura 2 muestra los sistemas coordenados utilizados en la modelización del sistema de medida, basado en el modelo "pinhole". Los planos perpendiculares a los ejes $Z_{P}$ y $Z_{C}$ poseen sistemas coordenados $(m, n)$ y $(u, v)$ en píxeles, y corresponden a la pantalla de cristal líquido del proyector y al plano de sensores de la cámara, respectivamente. Los orígenes de los sistemas coordenados corresponden a los centros de las pupilas de salida y entrada para el proyector y la cámara, respectivamente. Los planos se encuentran ubicados aproximadamente a las distancias focales. Una columna en la coordenada $m$ en píxeles, equivalente a la coordenada $x_{P}^{l}$ en $\mathrm{mm}$, genera un plano de luz, ver Figura 3, matemáticamente definido por la ecuación (1) en el sistema coordenado del proyector.

$$
f_{P} X_{P}-x_{P}^{l} Z_{P}=0 \text {. }
$$

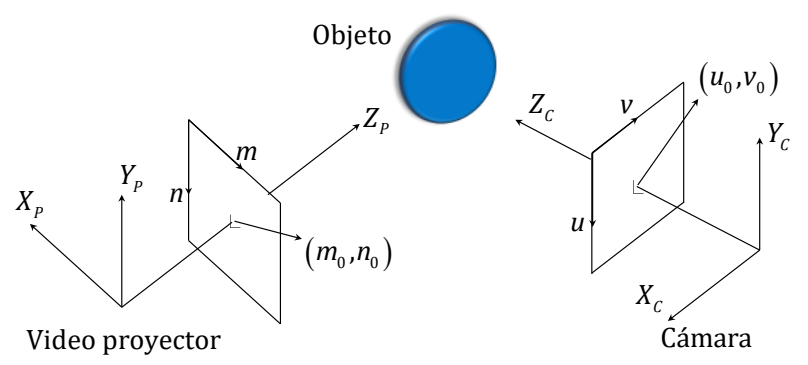

Fig.2. Sistemas coordenados utilizados para modelizar el sistema.

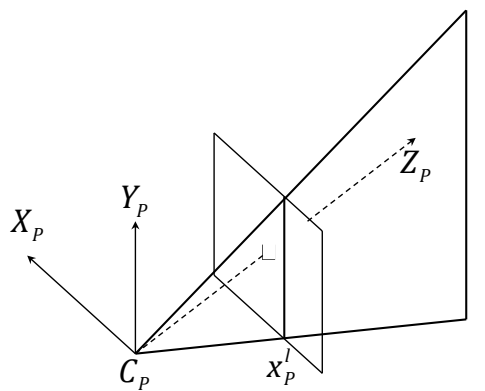

Fig.3. Plano de luz proyectado para la coordenada $m$. 
Los sistemas coordenados del proyector y de la cámara se pueden relacionar a partir de la transformación rígida definida por

$$
\left[\begin{array}{c}
X_{P} \\
Y_{P} \\
Z_{P}
\end{array}\right]=\left[\begin{array}{lll}
r_{C_{11}} & r_{C_{12}} & r_{C_{13}} \\
r_{C_{21}} & r_{C_{22}} & r_{C_{23}} \\
r_{C_{31}} & r_{C_{32}} & r_{C_{33}}
\end{array}\right]\left[\begin{array}{c}
X_{C} \\
Y_{C} \\
Z_{C}
\end{array}\right]+\left[\begin{array}{c}
t_{C_{1}} \\
t_{C_{2}} \\
t_{C_{3}}
\end{array}\right] .
$$

$r_{C_{i j}}$ y $t_{C_{i}}$ definen los elementos de la matriz de rotación y el vector de traslación entre los sistemas coordenados de la cámara $\left(X_{C}, Y_{C}, Z_{C}\right)$ y el proyector $\left(X_{P}, Y_{P}, Z_{P}\right)$. Resolviendo y realizando las sustituciones necesarias se logra determinar la ecuación del plano de luz de la coordenada $m$ en el sistema coordenado de la cámara

$$
\left[\begin{array}{c}
\alpha m \\
\alpha
\end{array}\right]=\left[\begin{array}{llll}
w_{11} & w_{12} & w_{13} & w_{14} \\
w_{21} & w_{22} & w_{23} & w_{24}
\end{array}\right]\left[\begin{array}{c}
X_{C} \\
Y_{C} \\
Z_{C} \\
1
\end{array}\right] .
$$

Donde $\alpha$ es un factor de escala que permite escribir la ecuación en forma matricial y los coeficientes $w_{i j}$ son función de los parámetros intrínsecos del proyector y los elementos $\mathbf{R}_{C}$ y $\mathbf{t}_{C}$. El procedimiento de calibración que se describe con más detalle en [11] consiste en determinar dichos coeficientes. Utilizando un patrón ajedrezado y la técnica propuesta por Zhang [12], se calculan los parámetros intrínsecos de la cámara; y mediante un procesamiento digital a la imagen, adquirida por la cámara, de un patrón de franjas que codifica en la fase las coordenadas $m$, se calculan las coordenadas $\left(u_{i}, v_{i}\right)$.

\section{Digitalización 3D}

Conociendo los parámetros intrínsecos de la cámara y la matriz $\mathbf{W}$ se calculan las coordenadas $X_{C}, Y_{C}, Z_{C}$, de un punto sobre la superficie del objeto como se muestra en la Figura 4. El video proyector emite un plano de luz correspondiente a la coordenada $m$ conocida. La cámara forma la imagen de la línea sobre la superficie de la córnea. Un tratamiento digital de la imagen permite calcular las coordenadas en píxeles del centro de la línea de luz. Para cada punto central de la línea de luz y usando los parámetros intrínsecos de la cámara se calcula la ecuación de la línea de visión que pasa por $p_{C}$ y $O_{C}$, ya que se conoce la columna $m$ de proyección, y según la ecuación (3) también se conoce la ecuación del plano de luz. A partir de la intersección entre la línea de visión de $p_{C}$ y el plano de luz se calculan las coordenadas $\left(X_{C}, Y_{C}, Z_{C}\right)$ del punto sobre la superficie. Este proceso se repite para todos los puntos centrales de la línea y para diferentes líneas proyectadas en el rango de valores de $m$ que cubran la superficie en estudio. De esta forma se obtiene una nube de puntos que corresponde a los puntos muestreados sobre la córnea.

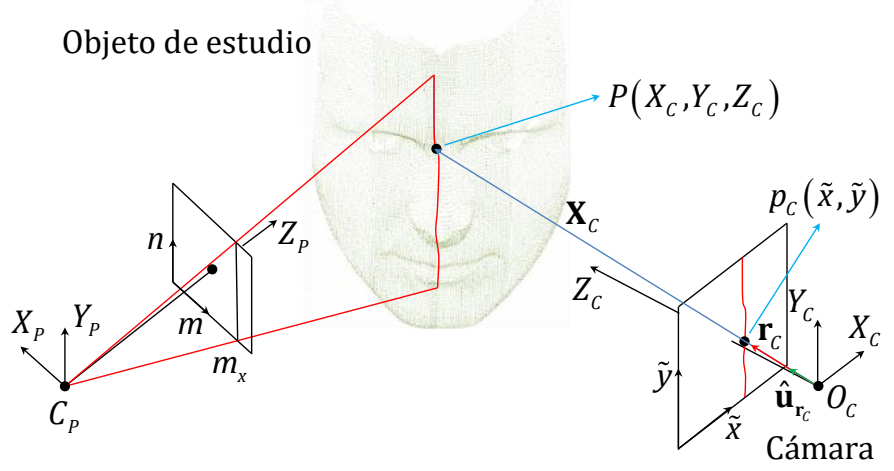

Fig.4. Procedimiento de digitalización 3D.

\section{Tratamiento digital a la imagen de la línea}

Para obtener las coordenadas del centro de la línea de luz adquirida por la cámara se realizó el procesamiento digital que se describe a continuación. Un procedimiento clásico de "skeleton" [13] permite obtener los puntos centrales de los objetos identificados en la imagen, previamente binarizada. La Figura 5 muestra en color rojo las líneas centrales obtenidas a partir del procedimiento mencionado. De las 
coordenadas de los puntos se evidencia que los que pertenecen a la línea de interés siguen aproximadamente una parábola. Se utilizó el método RANSAC (Random Sample Consensus), que es un método para determinar los parámetros de un modelo matemático, en nuestro caso una parábola, que se ajusta a un conjunto de datos. La línea azul de la Figura 5 muestra la parábola encontrada.

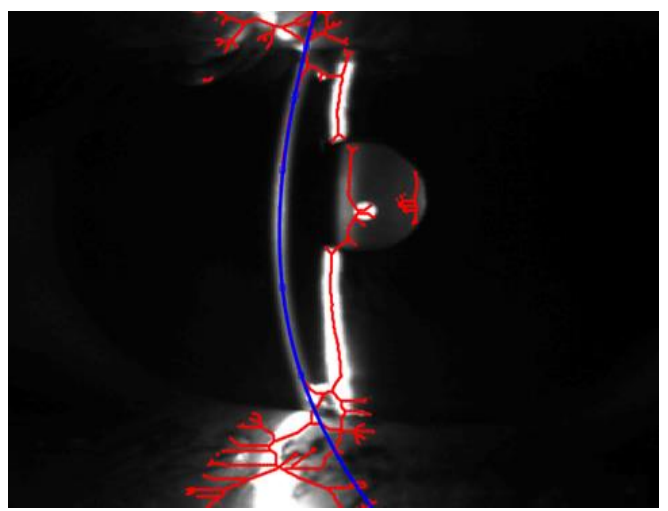

Fig.5. Líneas obtenidas a partir del procedimiento "skeleton" y parábola obtenida a partir del método RANSAC.

Un punto $(x, y)$ de la parábola permite extraer en dirección horizontal y a un ancho definido, que corresponde al ancho máximo de la línea de luz, un corte en intensidad sobre la imagen capturada por la cámara. Siguiendo un procedimiento de optimización con modelo Gaussiano, ver Figura 6, se calcula el centro de la campana, que corresponde a la posición central en dirección horizontal de la línea de luz para la coordenada " $y$ " de la parábola. Repitiendo el proceso para todos los puntos de la parábola se obtienen las coordenadas centrales de la línea de luz proyectada. Con el fin de reducir el tiempo de adquisición, se proyectaron simultáneamente tres líneas espaciadas de manera apropiada para evitar superposición de las mismas y por ende problemas en el procesamiento digital, ver Figura 7. La digitalización 3D se muestra en la Figura 8.

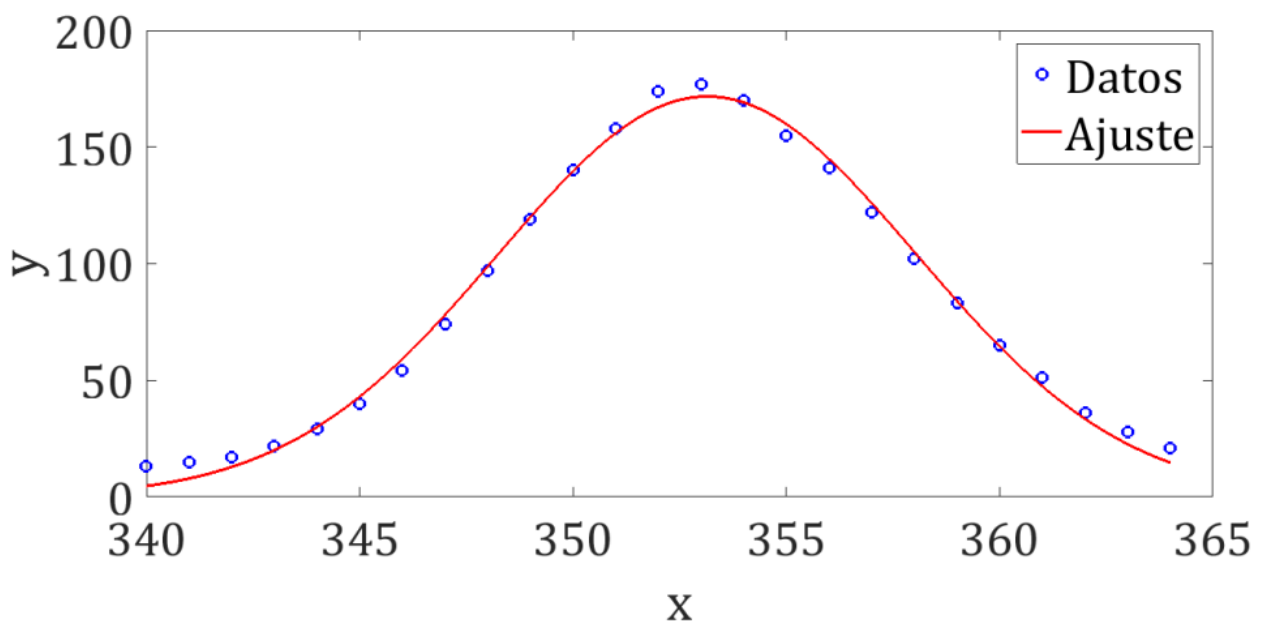

Fig.6. Ajuste Gaussiano de un corte en intensidad en dirección horizontal de la Fig 5 alrededor de la posición de la parábola encontrada con RANSAC para la posición vertical del corte: $\mathrm{x}$ en pixeles e y en Niveles de Gris. 


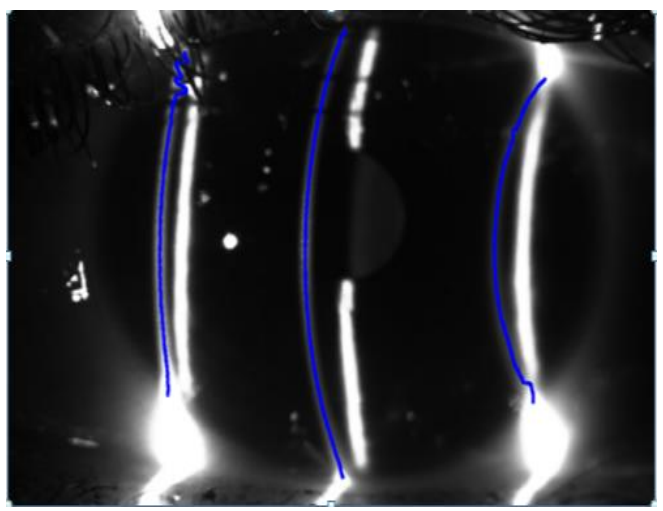

Fig.7. Líneas proyectadas sobre la córnea.

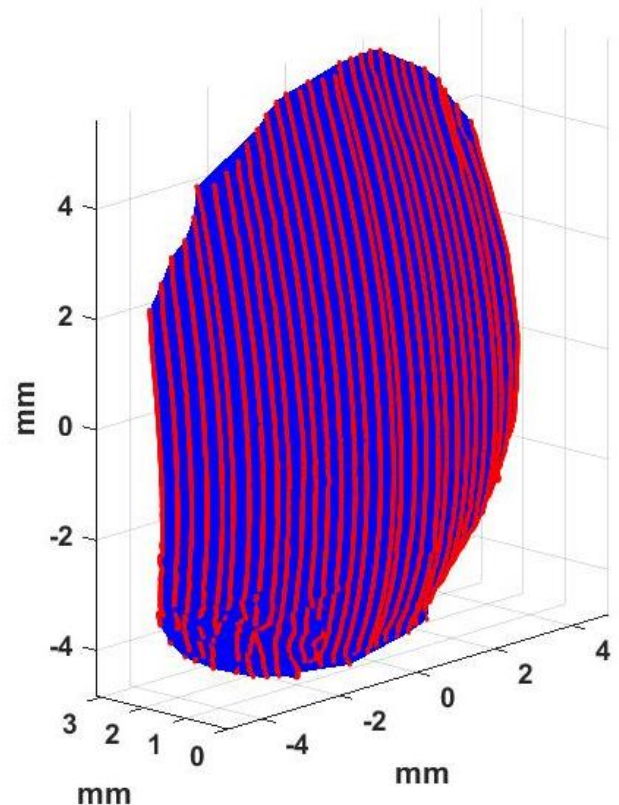

Fig. 8. Digitalización 3D de la superficie de la córnea.

\section{Conclusión}

En el presente trabajo se ha mostrado que la técnica de proyección de franjas permite digitalizar la superficie de la cara anterior de la córnea.

\section{Agradecimientos}

Los autores expresan su gratitud a la Universidad Santo Tomás, Seccional Bucaramanga y a la Universidad Industrial de Santander por garantizar la ejecución del presente proyecto, el cual hace parte de la IX convocatoria interna de la Universidad Santo Tomás bajo el código GIDTPOCBASICFACOP12016.

Partes de este trabajo fueron presentadas en la IX Reunión Iberoamericana de Óptica y XII Reunión Iberoamericana de Óptica, Láseres y Aplicaciones (RIAO/OPTILAS) realizada en noviembre de 2016 en Pucón, Chile. 